\title{
A micromachined surface stress sensor with electronic readout
}

\author{
Edwin T. Carlen, ${ }^{\text {a) }}$ Marc S. Weinberg, Angela M. Zapata, ${ }^{\text {b) }}$ and Jeffrey T. Borenstein \\ The Charles Stark Draper Laboratory, 555 Technology Square, Cambridge, Massachusetts 02139, USA
}

(Received 10 October 2007; accepted 11 December 2007; published online 11 January 2008)

\begin{abstract}
A micromachined surface stress sensor has been fabricated and integrated off chip with a low-noise, differential capacitance, electronic readout circuit. The differential capacitance signal is modulated with a high frequency carrier signal, and the output signal is synchronously demodulated and filtered resulting in a dc output voltage proportional to the change in differential surface stress. The differential surface stress change of the $\mathrm{Au}(111)$ coated silicon sensors due to chemisorbed alkanethiols is $\Delta \sigma_{s} \approx-0.42 \pm 0.0028 \mathrm{~N} \mathrm{~m}^{-1}$ for 1-dodecanethiol (DT) and $\Delta \sigma_{s} \approx$ $-0.14 \pm 0.0028 \mathrm{~N} \mathrm{~m}^{-1}$ for 1-butanethiol (BT). The estimated measurement resolution $(1 \mathrm{~Hz}$ bandwidth) is $\approx 0.12 \mathrm{mN} \mathrm{m}^{-1}$ (DT: $0.2 \mathrm{pg} \mathrm{mm}^{-2}$ and BT: $0.8 \mathrm{pg} \mathrm{m}^{-2}$ ) and as high as $\approx 3.82 \mu \mathrm{N} \mathrm{m}^{-1}$ (DT: $8 \mathrm{fg} \mathrm{mm}^{-2}$ and BT: $24 \mathrm{fg} \mathrm{mm}^{-2}$ ) with system optimization. () 2008 American Institute of Physics. [DOI: 10.1063/1.2830938]
\end{abstract}

\section{INTRODUCTION}

Surface stress is a critical factor for a wide variety of surface-related phenomena, such as surface reconstruction, nanoparticle shape transitions, surface alloying, surface diffusion, epitaxial growth, and self-assembled domain patterns. ${ }^{1,2}$ Macroscale structures have been used for many years to measure crystal response to surface stress ${ }^{3}$ and to characterize thin film properties of sputter deposited thin films. ${ }^{4}$ More recently, microfabricated silicon and silicon nitride cantilever beams have been used to measure the surface stress induced by molecular adsorption of biological materials on functionalized surfaces ${ }^{5,6}$ and alkanethiol adsorption on gold coated structures. ${ }^{7-10}$

Surface stress sensing of conformational changes of biomolecules selectively bound to a receptor layer may provide a viable alternative to resonant based techniques, such as quartz crystal microbalances and resonant cantilever beams,${ }^{11-13}$ for label-free biosensing. The surface stress sensing mechanism is fundamentally different from resonant mass sensing, where the latter detects a change in resonant frequency due to adsorption on the resonator. The detection resolution of the resonant mass sensors is typically reduced in a liquid medium due to the reduction of the resonator quality factor caused by increased viscous damping by the liquid. Techniques have been developed to improve this problem, ${ }^{14,15}$ however, with increased complexity to the sensor. Surface stress sensors detect low frequency deflection changes of mechanical structures due to differential surface stress changes of a sensing surface. Therefore, the resolution of the surface stress sensors is minimally affected by viscous damping. Other factors affecting the surface stress sensors in aqueous environments include plate deflections caused by the pressure head of the sample solution above the sensing

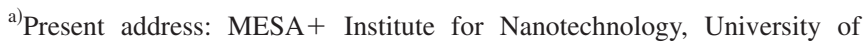
Twente, NL. Electronic mail: e.t.carlen@ewi.utwente.nl.

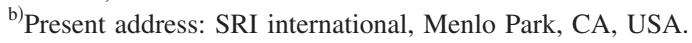

plate with height and density, the surface tension between the sample solution and the sensing surface, and ionic strength of the sample solution.

The surface stress sensors presented in this article are microfabricated from thin layers of single crystal silicon. The thin rectangular silicon layers are suspended with all edges clamped to a silicon substrate, therefore, physically separating the two plate surfaces; one surface is used for sensing and interfaces directly with the sample solution and the other surface is used for displacement detection. The purpose for isolating the sensing and detection surfaces is to facilitate the use of an electrical capacitance measurement to detect surface stress induced plate deflections. A low-noise differential capacitance measurement technique is used ${ }^{16}$ to measure the surface stress change of two different alkanethiol molecules, chemisorbed on the silicon sensing surfaces coated with a thin gold nucleation layer. The electronic detection technique provides a much more compact system package compared to the optical detection technique. Although the electronic detection technique can detect very small surface stress changes, for applications such as label-free biosensing, measuring absolute change in surface stress induced by molecular binding is not necessary; however, choosing a receptor layer with the appropriate functional group that generates a repeatable change in surface stress upon binding is essential such that the signal-to-noise ratio is as large as possible. Many questions still remain regarding the repeatability of surface stress sensing for different ligand-ligate systems and solution environments.

The microfabricated surface stress plate sensors presented here are advantageous, in our view, compared to cantilever beam structures in two important ways: (i) plate structures are more rigid than the beams with effective spring constants in the range of 50-100 $\mathrm{N} \mathrm{m}^{-1}$ and therefore can be easily functionalized and probed using commercially available printing techniques and (ii) the detection surface is physically isolated from the sensing surface and therefore can be easily adapted to other readout techniques in liquid 
(a)

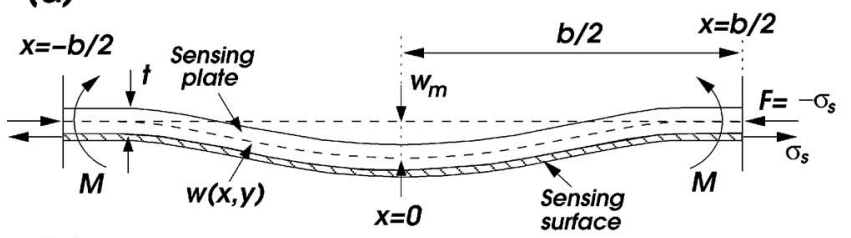

(c)

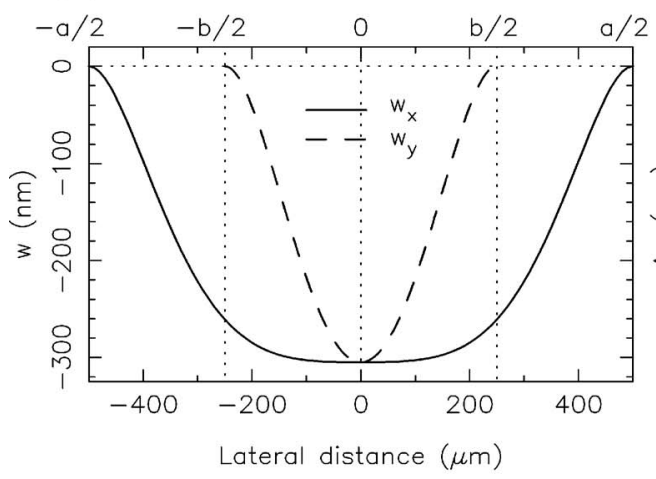

solutions, such as the low-noise differential capacitance measurement technique presented here. The microfabrication technology required to manufacture the plate sensors is more complex than the technology used to fabricate the cantilever beam sensors, where specialized release techniques are typically required for surface micromachined structures. However, surface micromachining fabrication technology is well established and provides a path to low-cost mass production of sensor structures.

Although the ratio of deflection to surface stress $\left(\eta=\Delta w / \Delta \sigma_{s}\right)$ for cantilever beams is typically larger than the plate structures by a factor of $\approx 10-100$ times, ${ }^{17}$ the electronic displacement detection resolution exceeds that of reported optical detection techniques ${ }^{18,19}$ by $\approx 10-100$ times, $^{20}$ suggesting that the plate structures with electronic readout are as sensitive as the cantilever beam-optical readout systems.

\section{THEORY AND DESIGN}

Surface stress has been previously described mathematically as $\sigma_{s_{i j}}=\delta_{i j} \gamma+\partial \gamma / \partial \varepsilon_{i j},{ }^{21-23}$ where the tensors can be represented as scalars for surfaces with lattice symmetry of three fold or larger [thin polycrystalline $\mathrm{Au}(111) \mathrm{hcp}$ films have a three fold lattice symmetry], $\sigma_{s}\left(\mathrm{~N} \mathrm{~m}^{-1}\right)$ is the surface stress, $\gamma\left(\mathrm{J} \mathrm{m}^{-2}\right)$ is the surface free energy, and $\varepsilon$ is the strain. The concept of surface stress implies that the surface stress performs work when straining a solid structure. In thin samples, surface stress can produce measurable elastic bending, such as the bending of the gold coated silicon plates due to the adsorption of an alkanethiol presented in this article.

\section{A. Plate bending}

From elasticity theory, assumptions from small plate deflections due to a uniform axial surface stress are used: (a) the plate material is homogeneous with uniform thickness $t$, (b) $t<b / 10$, where $b$ is the smallest plate dimension, (c) the maximum deflection $w_{m}<t / 2,{ }^{24,25}$ and (d) large deflection shearing forces and body forces are not considered. Figures (b)

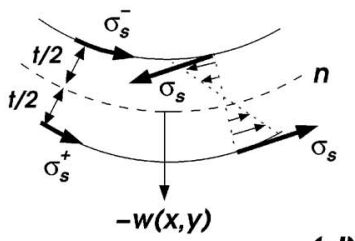

(d)

FIG. 1. (a) Dimensions and forces used to estimate plate bending, where $b$ is the plate width, $t$ is the plate thickness, and $\sigma_{s}$ is the differential surface stress (compressive in this case). (b) Plate bending due to $\sigma_{s}$. (c) Rectangular plate bending profiles in the $x$-direction $\left(w_{x}\right)$ and $y$-direction $\left(w_{y}\right)$ for $a=2 b$ and $w_{\delta}=305 \mathrm{~nm}$. (d) Center deflection $\Delta w$ as a function of $\Delta \sigma_{s}$ for several initial deflections $w_{\delta}$. 1(a) and 1(b) show dimensions and forces. Assuming uniform axial stress on the plate surface $\sigma_{s}^{+} \neq \sigma_{s}^{-}$, a differential surface stress $\sigma_{s}=\sigma_{s}^{+} \delta(z-t / 2)-\sigma_{s}^{-} \delta(z+t / 2),{ }^{26}$ where $\delta$ is the Dirac-delta function, has the effect of generating a stress couple of radial flexure bending moment $M$, shown in Fig. 1(b). This is equivalent to applying a force $F$ at the neutral surface $n$ thus generating moment $M$ at the clamped boundary such that the resultant force and moment on the edge are equal to zero. The bending moments are opposed by bulk moments of the plate represented as the plate flexural rigidity $D$. Since this approximation accurately predicts plate bending behavior away from the boundary areas, ${ }^{27}$ the deflections are measured at the plate center $(x=y=0)$.

The total plate deflection $w_{m}$ consists of two terms: one term due to an initial deflection $w_{\delta}$ and an additional deflection $\Delta w$ due to a radial surface force induced by the adsorption of the target molecule on the sensing surface. In practice, it is rare that suspended silicon plates are perfectly flat for a variety of reasons including imperfections in the silicon layer or surface, a thin stressed film on the plate surface, stress induced at boundary regions, adsorbed species on the surface, or deflections due to gravity. All suspended plates presented here have initial plate bending due primarily to the residual stress in the nucleation layer. ${ }^{28}$ Since $w_{\delta}$ is much larger than $\Delta w\left(w_{\delta} \sim 10 \times \Delta w\right)$, then $w_{\delta}$ must be considered when calculating the change in differential surface stress $\Delta \sigma_{s}$.

The total plate bending can, therefore, be determined by considering the deflection produced by the combination of a uniformly distributed lateral force $q\left(\mathrm{~N} \mathrm{~m}^{-2}\right)$, which is related to $w_{\delta}$, and a uniform in-plane force $F\left(\mathrm{~N} \mathrm{~m}^{-1}\right)$, which is related to $\sigma_{s}$. For a rectangular plate with clamped edges the estimated bending is $w(x, y)=\left(w_{\delta} / \Gamma_{0}\right)\left(1+\gamma \sigma_{s}\right) \Gamma(x, y)$ (Ref. 29) (see Appendix A), where $w_{\delta}$ is the initial center deflection, $\gamma$ is an estimated constant, $\Gamma_{0} \equiv \Gamma(0,0)$, and $\Gamma(x, y)$ is a shape function. Figure 1(c) shows an example of the rectangular plate bending in the $x$ and $y$ directions. The center deflection of the sensing plate is related to the differential surface stress change as $\Delta \sigma_{s} \approx \Delta w / w_{\delta} \gamma$, where $\Delta w$ 

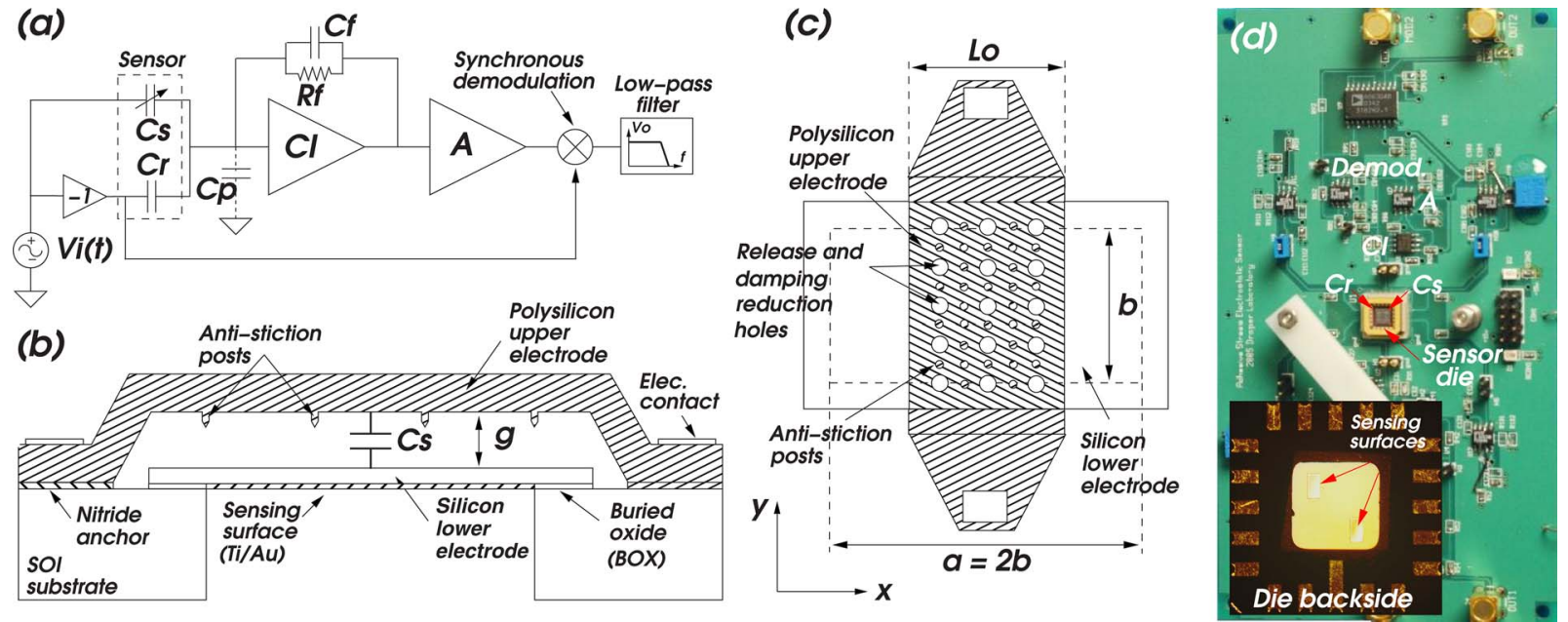

FIG. 2. (Color online) (a) Differential capacitance measurement circuit where CI is the charge integrator and $A$ is the amplifier. (b) Sensor cross section. (c) Top view of sensor with dimension labels. (d) Assembled electronics and sensor system.

$=w(0,0)_{t=t_{f}}-w(0,0)_{t=0}$ and $\Delta \sigma_{s}=\left(\sigma_{s}\right)_{t=t_{f}}-\left(\sigma_{s}\right)_{t=0}$ and assuming $\left(\sigma_{s}^{-}\right)_{t=t_{f}} \approx\left(\sigma_{s}^{-}\right)_{t=0}$. Figure 1(d) shows the dependence of $\Delta \sigma_{s}$ and $\Delta w$ on $w_{\delta}$.

Although the nucleation layer covers the entire plate surface in this article, surface stress induced deflections can be increased by partially covering the plate surface; therefore, the bending moment due to the edge of the nucleation layer adds to the total bending moment of the plate.

\section{B. Capacitance detection}

The capacitive electronic readout system, shown schematically in Fig. 2(a), uses a low-noise common-mode rejection configuration. The high frequency modulation technique is used to avoid $1 / f$ noise, electronic drifts, and line noise. The sense $C_{s}$ and reference $C_{r}$ capacitors are driven by the modulation signal $V_{i}(t)$. The differential capacitance is converted to a voltage with the charge integrating amplifier $(\mathrm{CI})$. The amplified modulated signal, containing phase and low frequency amplitude information, is synchronously demodulated and recovered as a dc signal at the output of the lowpass filter. The dynamic response of the measurement system must be balanced against the amount of attenuation from the low-pass filter required to adequately suppress the residual carrier components. The voltage output of the circuit is $\Delta V_{o} \propto-\left(\Delta C / C_{f}\right) V_{i}$, where $\Delta C$ is a function of $\Delta \sigma_{s}$.

The sensor is a conventional surface micromachined structure where the electrical capacitance is formed between the polysilicon and silicon layers. The device cross section is shown in Fig. 2(c). The reference capacitor is identical to the sense capacitor with the exception that the silicon layer is fixed to the substrate. The silicon nitride anchors the polysilicon layer to the substrate and provides electrical isolation. The buried oxide (BOX) layer anchors and isolates the silicon sense plate and the substrate. The antistiction posts prevent stiction between the polysilicon and silicon layers during the final release step of device fabrication due to surface tension effects during liquid drying.

The differential capacitance at the output of the CI consists of two terms, one due to the initial plate bending $C_{w_{\delta}}$ and one due to surface stress $C_{\Delta \sigma_{s}}$. Since the sensing plate has an initial deflection, the sensor has an inherent offset output voltage. The capacitance due to the surface stress change $C_{\Delta \sigma_{s}}$ is converted to a dc voltage and is used to estimate $\Delta \sigma_{s}$. The output voltage of the readout circuit is $\Delta V_{o} \approx V_{i} G\left(\Delta C / C_{f}\right) \cos (\phi)$, where $G$ is the total circuit gain at the modulation frequency and $\phi$ is the phase shift of the modulation signal (see Appendix B). Figure 2(d) shows the assembled electronics and sensor system, and the inset shows a sample die coated Au nucleation layer prior to testing.

\section{Detection limits}

Thermomechanical and electronic noise are the two dominant sources of noise limiting the performance of the surface stress sensor. Since the sensor is dynamically similar to a pressure sensor, the thermomechanical noise can be estimated with a simple second order harmonic oscillator with squeeze-film damping between the sensing plate and the polysilicon bridge. ${ }^{30}$ The thermomechanical limit on surface stress measurement is $\Delta \sigma_{s_{\mathrm{tm}}}$ $\approx \sqrt{4 k_{B} T R_{\mathrm{sf}}} / \gamma w_{\delta} k=2.84 \mu \mathrm{N} \mathrm{m}^{-1} \mathrm{~Hz}^{-1 / 2}$, where $k_{B}$ is Boltzmann's constant, $T=300 \mathrm{~K}$ is the ambient temperature, $k$ is the effective plate spring constant, and $R_{\mathrm{sf}}$ represents the squeeze-film damping. ${ }^{31}$

The minimum resolvable capacitance change the circuit can detect is $\Delta C \approx\left(V_{n} / V_{i}\right)\left(2 C_{s}+C_{f}+C_{p}\right)$, where $V_{n}$ is the noise voltage of the integration operational amplifier (SST441, Vishay Siliconix), $C_{f}$ is the feedback capacitor, and $C_{p}$ is a parasitic capacitance. The electronic noise limit on a surface stress measurement is $\Delta \sigma_{s_{e}}$ $\approx 0.12 \mathrm{mN} \mathrm{m}^{-1} \mathrm{~Hz}^{-1 / 2}$. Therefore, the total noise, due to both noise sources is $\Delta \sigma_{s_{t}}^{2}=\Delta \sigma_{s_{\mathrm{tm}}}^{2}+\Delta \sigma_{s_{e}}^{2}=0.12 \mathrm{mN} \mathrm{m}^{-1} \mathrm{~Hz}^{-1 / 2}$. Although the sensor is overdamped, the electronic noise dominates due to the large parasitic capacitance $C_{p}$. Improvements to the sensor structure indicate a surface stress resolution $\Delta \sigma_{s_{t}} \approx 3.82 \mu \mathrm{N} \mathrm{m}^{-1} \mathrm{~Hz}^{-1 / 2}$.

\section{MICROFABRICATION}

The microsensor has been fabricated using a conventional surface micromachining process ${ }^{32}$ with silicon-on- 

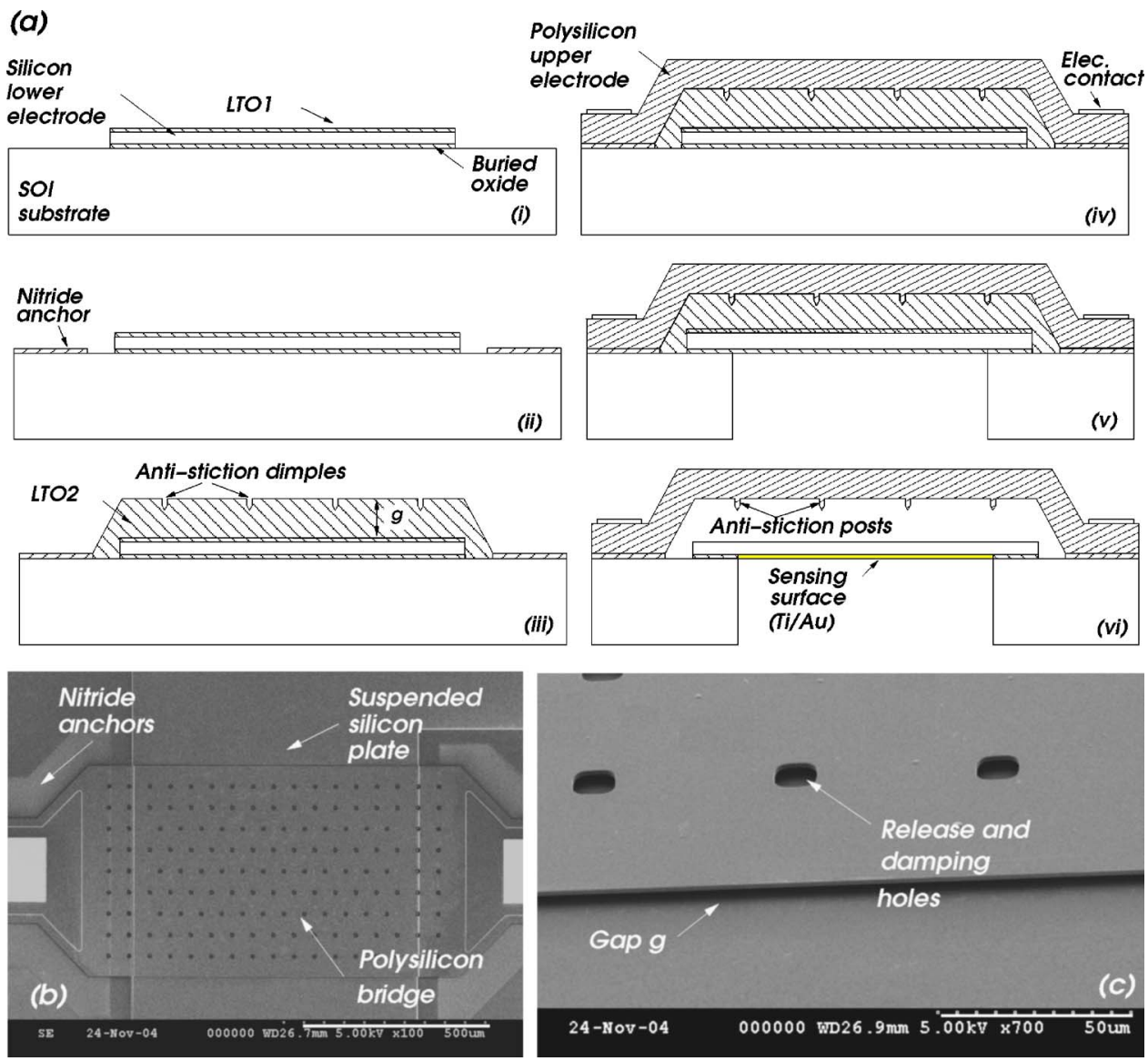

FIG. 3. (Color online) (a) Simplified sensor microfabrication process flow. (b) Top view scanning electron microscope (SEM) of released sensor structure. (c) SEM of released capacitor structure.

insulator (SOI) substrates and deep reactive ion etching (DRIE) of the bulk silicon substrates. The entire process uses seven lithography steps. Figure 3(a) highlights the essential aspects of the microfabrication process. A $1 \mu \mathrm{m}$ thick low temperature oxide (LTO1) etch mask film is first deposited on the SOI substrates in a low-pressure chemical vapor deposition (LPCVD) system, followed by contact lithography patterning and reactive ion etching (RIE) thus defining the sense plate. The BOX layer is then removed, as shown in Fig. $3(\mathrm{a}, \mathrm{i})$. Next, the $1.0 \mu \mathrm{m}$ thick low-stress LPCVD nitride anchor layer is deposited, patterned, and RIE, thus defining the upper electrode anchor layer, shown in Fig. 3(a,ii). The remaining LTO1 mask layer is removed in a dilute hydrofluoric acid (HF) solution. A $3 \mu \mathrm{m}$ thick low temperature LPCVD oxide layer (LTO2) is deposited defining the separation gap g. Dimple patterns are first patterned and RIE in LTO2, $1 \mu \mathrm{m}$ deep. The LTO2 layer is then patterned and RIE to open contact holes to the nitride layer, shown in Fig. 3(a,iii). The $4 \mu \mathrm{m}$ thick LPCVD low-stress polysilicon layer is deposited next and doped. ${ }^{33}$ The polysilicon layer is then patterned and RIE thus opening the release holes (and damping reduction holes) and defining the upper polysilicon plate structure. Electrical contact areas are opened by patterning and RIE the LTO2 layer, followed by metallization $(30 \mathrm{~nm} \mathrm{Cr} / 500 \mathrm{~nm} \mathrm{Au})$ by sputtering and lift-off, shown in Fig. 3(a,iv). Next, the back side of the substrate is lithographically patterned and aligned to the device layer, and the silicon handle layer is removed using silicon DRIE, shown in Fig. 3(a,v). The remaining oxide layers are then removed in a 3:1 HF: $\mathrm{H}_{2} \mathrm{O}$ solution, shown Fig. 3(a,vi).

\section{EXPERIMENTS AND DISCUSSION}

Self-assembling alkanethiol monolayers on $\mathrm{Au}(111)$ nucleation layers are used for surface stress measurements. Alkanethiols are highly ordered and stable molecular monolayers that spontaneously organize on Au surfaces. ${ }^{34-36}$ The high affinity of thiols for noble metal surfaces provides an attractive medium to generate well-defined organic surfaces with a wide range of chemical functionalities displayed at the sensing interface.

The sensing surfaces are first sputter coated with the $\mathrm{Ti} / \mathrm{Au}$ nucleation layer and mounted with the readout electronics on the printed circuit (PC) board, the assembly is then mounted in the test fixture shown schematically in Fig. 4(a). Figure 4(b) shows x-ray diffraction data from sample $\mathrm{Ti} / \mathrm{Au}$ sputtered films. ${ }^{37}$ The sensor baseline is first established for $60 \mathrm{~s}$ before exposing the sensing surface to the test vapor. The $\mathrm{Au}(111)$ coated sensing surface is then exposed to the alkanethiol vapor from a large sealed reservoir of liquid $\approx 1.3 \mathrm{~mL}$ for a time of $300 \mathrm{~s}$. Figure $4(\mathrm{c})$ shows the sensor response of a test device. Prior to exposure, the offset voltage of $\approx 500 \mathrm{mV}$ is consistent with the capacitance change due to initial plate bending of $w_{\delta} \approx 305 \mathrm{~nm}$. The 

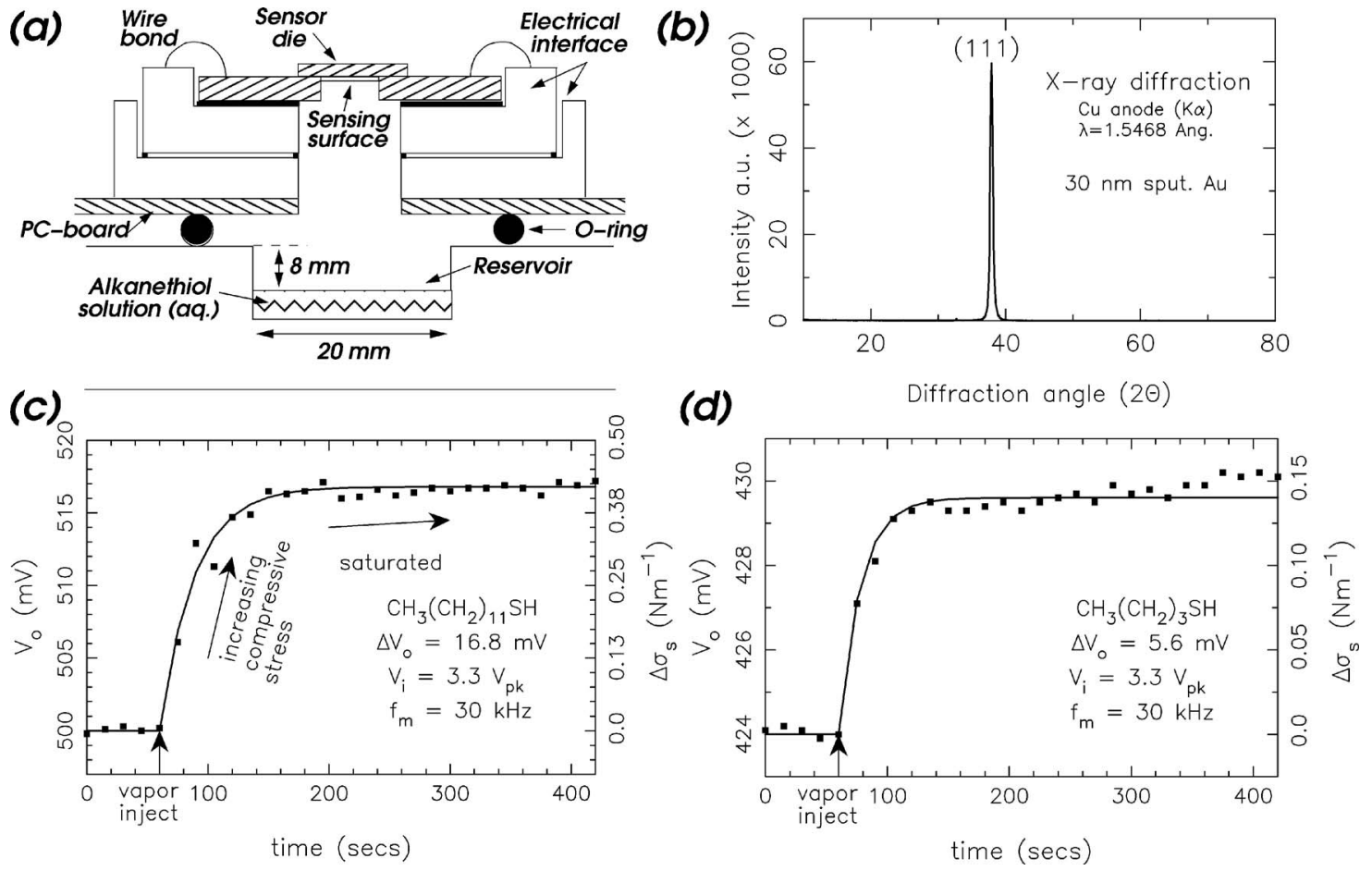

FIG. 4. (a) Schematic of test fixture. (b) X-ray diffraction scan of $30 \mathrm{~nm}$ sputtered Au layer (with $8 \mathrm{~nm}$ Ti adhesion layer). (c) Measured 1-dodecanethiol response where $\Delta \sigma_{s}$ plotted with $w_{\delta}=305 \mathrm{~nm}$ and $V_{o}$ plotted with $\kappa=0.056 \mathrm{~s}^{-1}$ (d) Measured 1-butanethiol response where $\Delta \sigma_{s}$ calculated with $w_{\delta}=312 \mathrm{~nm}$ and $V_{o}$ calculated with $\kappa=0.035 \mathrm{~s}^{-1}$. Solid lines of (c) and (d) calculated from the Langmuir isotherm relationship $V_{o}=\Delta V_{o}[1-\exp (-\kappa t)]$. All calculations use $g=3.2 \mu \mathrm{m}$ and $b=480 \mu \mathrm{m}$.

exposure of the sensing surface at $t=60 \mathrm{~s}$ to vapor phase (as received) 1-dodecanethiol $\left[\mathrm{CH}_{3}-\left(\mathrm{CH}_{2}\right)_{11}-\mathrm{SH}, \geqslant 98 \%\right.$ Aldrich No. 471364] results in a surface stress change $\Delta \sigma_{s} \approx-0.42 \pm 0.0028 \mathrm{~N} \mathrm{~m}^{-1}$, smaller than the value of $-0.72 \pm 0.02 \mathrm{~N} \mathrm{~m}^{-1}$ reported by Carlen et al. ${ }^{28}$ larger than the reported value of $-0.2 \mathrm{~N} \mathrm{~m}^{-1}$ by Berger et al., ${ }^{8}$ but close to the measured value of $-0.52 \pm 0.01 \mathrm{~N} \mathrm{~m}^{-1}$ reported by Godin et al. ${ }^{10}$ Figure 4(d) shows the sensor response of a different test device. The offset voltage of $\approx 420 \mathrm{mV}$ is consistent with $w_{\delta} \approx 312 \mathrm{~nm}$. The sensing surface exposed, at $t=60 \mathrm{~s}$, to 1-butanethiol (used as received) $\left[\mathrm{CH}_{3}-\left(\mathrm{CH}_{2}\right)_{3}-\mathrm{SH}\right.$, Aldrich No. 240966] vapor results in a surface stress change $\Delta \sigma_{s} \approx-0.14 \mathrm{~N} \mathrm{~m}^{-1}$, larger than the value of $-0.08 \mathrm{~N} \mathrm{~m}^{-1}$ reported by Berger et al. ${ }^{8}$

The microfabricated surface stress plate sensors presented here demonstrate important improvements to cantilever beam structures. Since the detection surface is physically isolated from the sensing surface, the low-noise differential capacitance measurement technique can be used which is more compact compared to conventional optical readout systems while providing comparable sensitivity. Additionally, the material to be sensed is confined to a single surface, thus eliminating the possibility of attachment to undesired surfaces.

\section{ACKNOWLEDGMENTS}

The authors thank The Charles Stark Draper Laboratory for research funding, Connie Cardoso, Mert Prince, and Manuela Healey for fabrication assistance, John Lachapelle for building the electronics, and Caroline Kondoleon for sensor packaging.

\section{APPENDIX A: PLATE BENDING CALCULATION}

A numerical solution of $\nabla^{4} w \pm(F / D) \nabla^{2} w=q / D$ with boundary conditions $(w)_{x, y= \pm a / 2, b / 2}=0$ and $(\partial w / \partial x)_{x=0, \pm a / 2}$ $=(\partial w / \partial y)_{y=0, \pm b / 2}=0$, for a particular force $F$, is $w(x, y)$ $=\left(q b^{4} / D\right) \Lambda(x, y),{ }^{29}$ where $q$ is a uniform lateral pressure, $b$ is the plate width (plate length $a=2 b$ ), $D=E t^{3}\left(1-\nu^{2}\right)^{-1} / 12, E$ is the elasticity modulus and $\nu$ is the Poisson ratio, and $\Lambda(x, y)$ is shape function defined as $\Lambda(x, y)=\left[1-v_{1} \Psi\left(2 \kappa_{1} y / b\right)-v_{2} \Upsilon\left(2 \kappa_{1} y / b\right)\right] \cdot\left[1+v_{3} \Psi\left(2 \kappa_{2} x / a\right)\right.$ $\left.-v_{4} \Upsilon\left(2 \kappa_{2} x / a\right)\right]$, where $\Psi(x)=\cos (x) \cosh (x)$ and $Y(x)$ $=\sin (x) \sinh (x)$. Since, in this case, it was found that the functional dependence of $w(x, y)$ on $F$ is approximately linear in the range $-1.5 \leqslant F \leqslant+1.5 \mathrm{~N} \mathrm{~m}^{-1}$, then the plate deflection is estimated as $w(x, y)=\left(q b^{4} / D\right)\left(1+\gamma \sigma_{s}\right) \Gamma(x, y)$, where $F=-\sigma_{s}, \gamma$ is a fitting constant, and $\Gamma$ is the shape function when $F=0, \Gamma(x, y)=\Lambda(x, y)_{F=0}$.

Since the initial center deflection, defined as $w_{\delta}$, is a measurable quantity, $q$ is determined when $\sigma_{s}=0$ at the plate center $(x=y=0)$, therefore $q=w_{\delta} D / b^{4} \Gamma_{0}$, where $\Gamma_{0} \equiv \Gamma(0,0)$. For all calculations, the following constants and dimensions are used, unless specified otherwise, $b=500 \mu \mathrm{m}, t=2 \mu \mathrm{m}, E=150 \mathrm{GPa}, \nu=0.2, \Gamma_{0}=0.515611$, $\gamma=-0.065776, v_{1}=0.51441, v_{2}=0.430155, v_{3}=0.061892$, $v_{4}=0.0069447, \kappa_{1}=1.47201$, and $\kappa_{2}=3.81478$.

\section{APPENDIX B: ELECTRICAL CAPACITANCE CALCULATION}

The electrical capacitance of a perfectly flat sensing plate is $C_{o}=\varepsilon_{0} b L_{o} / g$, where $b, L_{o}$, and $g$ are shown in Figs. 2(b) and 2(c). The capacitance due to the initial deflection is $C_{w_{\delta}}=\int_{-b / 2}^{b / 2} \int_{-a / 4}^{a / 4}\left\{\varepsilon_{0} /\left[g \pm\left(w_{\delta} / \Gamma_{0}\right) \Gamma(x, y)\right]\right\} d x d y$ 
$\approx \int_{-b / 2}^{b / 2}\left\{L_{o} \varepsilon_{0} /\left[g \pm\left(w_{\delta} / \Gamma_{0}\right) f(y)\right]\right\} d y$, where $f(y)$ is a six term expansion of $w(0, y)$. The capacitance change due to the surface stress change is $C_{\Delta \sigma_{s}} \approx \int_{-b / 2}^{b / 2}\left\{L_{o} /\left[g \pm\left(w_{\delta} / \Gamma_{0}\right)(1\right.\right.$ $\left.\left.\left.+\gamma \Delta \sigma_{s}\right) f(y)\right]\right\} d y$. The capacitance resulting from the surface stress change only is $\Delta C_{\Delta \sigma_{s}}=C_{w \delta}-C_{\Delta \sigma_{s}}$. The readout circuit is designed for a full-scale dynamic range for $\Delta \sigma_{s}$ of $3 \mathrm{~N} \mathrm{~m}^{-1}$ resulting in a maximum deflection of $\approx 60 \mathrm{~nm}$. A $3 \mu \mathrm{m}$ separation gap results in $\approx 1.96 \%$ nonlinearity in the electronics measurement. The scale factor is (SF) $\approx 25 \mathrm{mV} / \mathrm{N} \mathrm{m}^{-1}$. The modulation frequency is $f_{m}=30 \mathrm{kHz}$, which is far from the mechanical resonant frequency of the sensor $f_{r} \approx 80 \mathrm{kHz}$. For all calculations $G=8.8, \phi=1^{\circ}$, $C_{s}=1 \mathrm{pF}, \quad C_{f}=1.4 \mathrm{pF}, \quad C_{p}=150 \mathrm{pF}, \quad V_{n}=5 \mathrm{nV} \mathrm{Hz}^{-1 / 2}$, $w_{\delta}=305 \mathrm{~nm}$, and $g=3.2 \mu \mathrm{m}$.

${ }^{1}$ H. Ibach, Surf. Sci. Rep. 29, 193 (1997).

${ }^{2}$ D. Sander, Curr. Opin. Solid State Mater. Sci. 7, 51 (2003).

${ }^{3}$ J. Cahn and R. Hanneman, Surf. Sci. 1, 387 (1964).

${ }^{4}$ R. Hoffman, Physics of Thin Films, edited by George Hass and R. E. Thun (Academic, New York, 1966).

${ }^{5}$ A. Moulin, S. O'Shea, R. Badley, P. Doyle, and M. Welland, Langmuir 15, 8776 (1999).

${ }^{6}$ G. Wu, R. Dat, K. Hansen, T. Thundat, R. Cote, and A. Majumdar, Nat. Biotechnol. 19, 856 (2001).

${ }^{7}$ H.-J. Butt, J. Colloid Interface Sci. 180, 251 (1996).

${ }^{8}$ R. Berger, E. Delamarche, H. Lang, C. Gerber, J. Gimzewski, E. Meyer, and H.-J. Güntherodt, Science 276, 2021 (1997).

${ }^{9}$ R. Raiteri, H.-J. Butt, and M. Grattarola, Scanning Microsc. 12, 243 (1998).

${ }^{10}$ M. Godin, P. Williams, V. Tabard-Cossa, O. Laroche, L. Beaulieu, R. Lennox, and P. Grutter, Langmuir 20, 7090 (2004).

${ }^{11}$ K. Marx, Biomacromolecules 4, 1099 (2003).

${ }^{12}$ T. Thundat, E. Wachter, S. Sharp, and R. Warmack, Appl. Phys. Lett. 66, 1695 (1995).

${ }^{13}$ B. Ilic, D. Czaplewski, H. Craighead, P. Neuzil, C. Campagnolo, and C. Batt, Appl. Phys. Lett. 77, 450 (2000).

${ }^{14} \mathrm{~J}$. Tamayo, A. Humphris, A. Malloy, and M. Miles, Ultramicroscopy 86, 167 (2001).

${ }^{15}$ T. Burg and S. Manalis, Appl. Phys. Lett. 83, 2698 (2003).

${ }^{16} \mathrm{~A}$ commercially available sensor reports a displacement resolution of $\approx 210 \mathrm{fm}$ (1 Hz bandwidth) (Ref. 38).

${ }^{17}$ Compared to cantilever beam dimensions from (Refs. 5-8 and 39) and plate dimensions presented here.

${ }^{18}$ D. Rugar, H. Mamin, and P. Guethner, Appl. Phys. Lett. 55, 2588 (1989).
${ }^{19}$ G. Yaralioglu, A. Atalar, S. Manalis, and C. Quate, J. Appl. Phys. 83, 7405 (1998).

${ }^{20} \mathrm{~A}$ displacement resolution of $0.001 \mathrm{~nm}$ was reported (Ref. 18) using an optical interferometric technique; however, $0.01 \mathrm{~nm}$ (Ref. 19) is more common.

${ }^{21}$ R. Shuttleworth, Proc. Phys. Soc., London, Sect. A 63, 444 (1950).

${ }^{22}$ J. Vermaak, C. Mays, and D. Kuhlmann-Wilsdorf, Surf. Sci. 12, 128 (1968).

${ }^{23}$ P. Couchman, W. Jesser, and D. Kuhlmann-Wilsdorf, Surf. Sci. 33, 429 (1972).

${ }^{24}$ S. Timoshenko, Theory of Plates and Shells (McGraw-Hill, New York, 1959).

${ }^{25}$ For all devices tested (a) nominal plate thickness $t=2 \mu \mathrm{m}$, (b) $t=b / 250$, and (c) $w_{m} \leqslant t / 4$. Szilard (Ref. 40) recommends $t / 10 \leqslant w_{m} \leqslant t / 5$.

${ }^{26}$ Defined as tension $\sigma_{s}>0$ and compression $\sigma_{s}<0$.

${ }^{27} \mathrm{~S}$. Timoshenko and J. Goodier, Theory of Elasticity, 3rd ed. (McGrawHill, New York, 1970).

${ }^{28}$ E. Carlen, M. Weinberg, C. Dubé, A. Zapata, and J. Borenstein, Appl. Phys. Lett. 89, 173123 (2006).

${ }^{29}$ C. Chang and H. Conway, J. Appl. Mech. 19, 179 (1952).

${ }^{30}$ T. Gabrielson, IEEE Trans. Electron Devices 40, 903 (1993).

${ }^{31}$ The surface stress signal is defined as $\left|Z_{s}\right|=|\Delta w|$ and the noise signal is $\left|Z_{n}\right|=\sqrt{4 k_{B} T R_{\mathrm{sf}}} / k$. To determine the detection limit, set $\left|Z_{s} / Z_{n}\right|=1$, and calculate $\Delta \sigma_{s_{\mathrm{tm}}}$, where $|\Delta w|=\left|\Delta \sigma_{s_{\mathrm{tm}}} \gamma w_{\delta}\right|$. The effective spring constant is calculated from Hooke's law $k=\left|F_{p} / \Delta z\right|$, where $F_{p}$ is a point force applied to the plate center. For a rectangular plate, $k=D / c_{1} b^{2}$, where $c_{1}=0.00722$ for $a / b=2$ (Ref. 40), resulting in $k \approx 58 \mathrm{~N} \mathrm{~m}^{-1}$. The estimated squeeze-film damping is $R_{\mathrm{sf}} \approx 0.33 \mathrm{mN} \mathrm{s} \mathrm{m}^{-1}$ (Refs. 41 and 42 ).

${ }^{32}$ D. Koester, A. Cowen, R. Mahadevan, M. Stonefield, and B. Hardy, PolyMUMPS Design Handbook Rev. 10 (2003).

${ }^{33}$ The polysilicon is doped using boron ion implantation $\left({ }^{11} \mathrm{~B}^{+}\right.$, $\phi=10^{16} \mathrm{~cm}^{-2}, E=150 \mathrm{keV}$, angle $\left.=7^{\circ}\right)$ followed by annealing at $1000{ }^{\circ} \mathrm{C}$ for $5 \mathrm{~h}$ in a $100 \% \mathrm{~N}_{2}$ environment.

${ }^{34}$ R. Nuzzo and D. Allara, J. Am. Chem. Soc. 105, 4481 (1983).

${ }^{35}$ R. Nuzzo, B. Zegarski, and L. Dubois, J. Am. Chem. Soc. 109, 733 (1987).

${ }^{36}$ C. Bains, E. Troughton, Y.-T. Tao, J. Evall, G. Whitesides, and R. Nuzzo, J. Am. Chem. Soc. 111, 321 (1989).

${ }^{37}$ Peak occurs at $2 \theta=37.94^{\circ}$ corresponding to (111) direction for Au.

${ }^{38}$ J. Doscher, Analog Dialogue 33, 27 (1999).

${ }^{39}$ J. Pei, F. Tian, and T. Thundat, Anal. Chem. 76, 292 (2004).

${ }^{40} \mathrm{R}$. Szilard, Theory and Analysis of Plates: Classical and Numerical Methods (Prentice-Hall, Englewood Cliffs, 1989).

${ }^{41}$ J. Bergqvist, F. Rudolf, J. Maisana, F. Parodi, and M. Rossi, Digest of Technical Papers of the 1991 International Conference on Solid-State Sensors and Actuators, 24-27, pp. 266-269 (1991) .

${ }^{42}$ P. Kwok, M. Weinberg, and K. Breuer, J. Microelectromech. Syst. 14, 770 (2005). 